Article

\title{
Enhanced Quality of Service of Cell-Edge User by Extending Modified Largest Weighted Delay First Algorithm in LTE Networks
}

\author{
Hasibur Rashid Chayon ${ }^{1}$, Kaharudin Bin Dimyati ${ }^{1}$, Harikrishnan Ramiah ${ }^{1, *}$ and \\ Ahmed Wasif Reza ${ }^{2}$ \\ 1 Department of Electrical Engineering, Faculty of Engineering, University of Malaya, Kuala Lumpur 50603, \\ Malaysia; hasibur.rashid@siswa.um.edu.my (H.R.C.); kaharudin@um.edu.my (K.B.D.) \\ 2 Department of Computer Science \& Engineering, Faculty of Science \& Engineering, East West University, \\ Dhaka 1212, Bangladesh; wasif@ewubd.edu \\ * Correspondence: hrkhari@um.edu.my; Tel.: +603-7967-5262
}

Academic Editor: Angel Garrido

Received: 21 March 2017; Accepted: 29 May 2017; Published: 31 May 2017

\begin{abstract}
Long Term Evolution (LTE) is the prominent technology in Fourth Generation (4G) communication standards, which provides higher throughput and better Quality of Service (QoS) to all users. However, users in the cell-edge area are receiving comparatively low QoS due to the distance from eNodeB (eNB) and bad channel conditions. The Conventional Modified Largest Weighted Delay First (MLWDF) algorithm is unable to resolve this issue, as it does not consider the location of the user. This paper proposes an extended MLWDF (EMLWDF) downlink scheduling algorithm to provide better services to the cell-edge user as well as to the cell-center user. The proposed algorithm divides the eNB cell area into inner and outer regions. It includes the distance of the user from attached eNB, received Signal to Interference plus Noise Ratio (SINR) and error probability into the original algorithm. The simulated results are compared with other well-known algorithms and the comparison shows that the proposed algorithm enhances overall $56.23 \%$ of cell-edge user throughput and significantly improves the average user throughput, fairness index, and spectral efficiency.
\end{abstract}

Keywords: LTE; scheduling algorithm; MLWDF; cell-edge user; SINR

\section{Introduction}

The Third Generation Partnership Project (3GPP) introduced Long Term Evolution (LTE) Fourth Generation (4G) technology in Release-8 (Rel-8). It is based on Orthogonal Frequency Division Multiple Access (OFDMA). After doing a significant improvement in Rel-9, LTE provides higher data rates and better Quality of Service (QoS) than Third Generation (3G) mobile technology. The QoS is better in the cell-center area as users are close to the eNodeB (eNB), which strengthens the received signal. However, users in the cell-edge area are experiencing low data rates and poor QoS due to low received signal, high interference from adjacent eNBs and various fading effects [1].

The packet scheduler implemented in eNB plays a vital role to enhance the QoS. This can be done by allocating time-frequency blocks known as Resource Blocks (RBs) to different users according to their current situations and priorities [2]. RBs of the OFDMA system is defined by the time and frequency domains in the $x$ - and $y$-axes, respectively. A $10 \mathrm{~ms}$ long radio frame in the time domain is divided into $10 \mathrm{sub}$-frames each with $1 \mathrm{~ms}$ duration. Each sub-frame consists of two equal-sized time slots with duration of $0.5 \mathrm{~ms}$. Each slot is composed of six or seven OFDMA symbols for extended and normal cyclic prefix respectively. In the frequency domain, one RB has 12 consecutive subcarriers with in total $180 \mathrm{kHz}$, where each subcarrier has $15 \mathrm{kHz}$ bandwidth [3,4]. Figure 1 shows the basic RB structure of LTE with a normal cyclic prefix. 


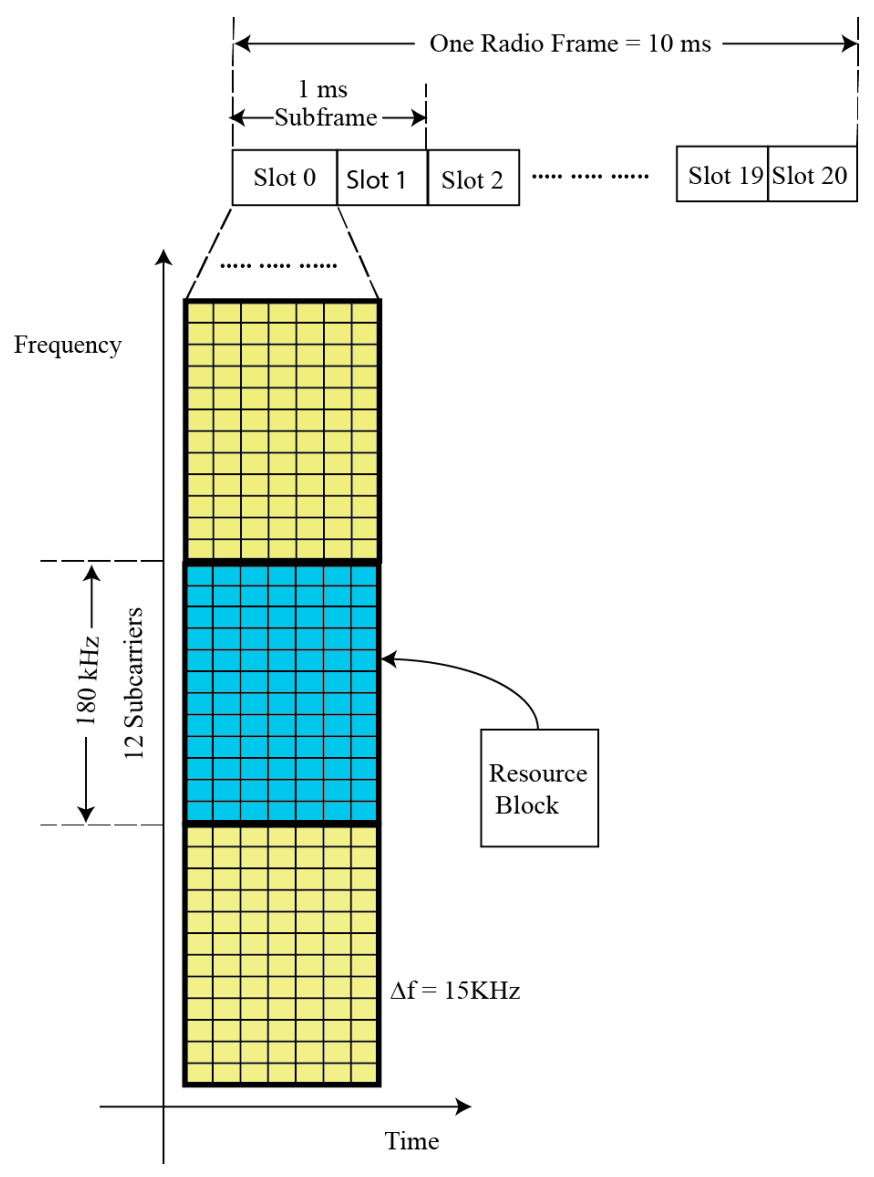

Figure 1. Time-frequency Resource block (RB) structure of Long Term Evolution (LTE).

The packet scheduler in the LTE system calculates the metric of all users according to the corresponding algorithm. It considers various factors, such as instantaneous and previous throughput, spectral efficiency, head of line (HOL) packet delay, the number of packets waiting in the queue and several scheduling constants. The user with higher metric value will be in front of the queue to get the service. An efficient packet scheduler can reduce the traffic congestion in the network. Different downlink scheduling algorithms have different approaches to determine the user priority. Proportional fairness (PF) is the basic scheduler [5], which is based on prioritizing the user with maximum metrics from the ratio of the instantaneous and previous data rates. This scheduling algorithm does not consider any kind of packet delay or queue length. Therefore, real-time data are experiencing low QoS and high rate of packet loss. The Modified Largest Weighted Delay First (MLWDF) algorithm ensures ideal throughput to the system and maintains better fairness to all users than other algorithms [6-8]. This scheduler largely depends on QoS and packet delay. The throughput optimal MLWDF algorithm has less complexity among all other scheduling algorithms and it considers the real-time traffic. The primary goals of this scheduler are to provide better QoS, to enhance the spectral efficiency and to provide good fairness to the user. Other scheduling algorithms such as exponential proportional fairness (EXP/PF), exponential rule (EXP/Rule) and Logarithmic rule (LOG/Rule) are comparatively more complex to implement [9]. Therefore, this paper extends the MLWDF algorithm to provide services.

PF and basic MLWDF algorithms are considered in the simulation process to compare the performance with the proposed extended MLWDF (EMLWDF) algorithm. This proposed algorithm gives more priority to the cell-edge users by considering the distance between user and eNB, downlink Signal to Interference plus Noise Ratio (SINR) and packet delay while maintaining the cell performance. 
The remaining paper is organized as follows. Section 2 describes the related work followed by the system model of the proposed algorithm in Section 3. Section 4 has results and discussion, Section 5 describes limitations and future work and conclusions are presented in Section 6.

\section{Related Works}

Interference from the neighboring cell significantly affects the cell-edge user as well as the system performance. Therefore, inter-cell interference (ICI) has been studied previously to overcome the problems of poor QoS and low throughput of the cell-edge users $[10,11]$. The authors consider the soft frequency reuse (SFR) technique, which is a form of inter-cell interference coordination (ICIC) for LTE systems in [12-14]. A decentralized SFR scheduling algorithm with low complexity is proposed in [12], which incorporates user classification and balance fairness and throughput among all users. Two types of frequency bands are used in a cell, namely, cell-center band (CCB) and cell-edge band (CEB). Moreover, users are also separated into schedules in CCB groups and in CEB groups. The users with higher interference from the adjacent cells are defined as the cell-edge users (CEUs) and the rest of the users are the cell-center users (CCUs). In [13], the authors proposed a softer frequency reuse (SerFR) method, which is the modified version of SFR. In this method, the frequency reuse factor (FRF) of cell edge and cell center is one. High power frequency bands are allocated to the cell edge users and low power bands are allocated to the cell center users. In [14], an adaptive SFR is considered by altering the transmit power and the number of major subcarriers for individual cells based on the traffic load. This method decentralizes the resource allocation decision by sharing the subcarrier, transmit power and rate information with the neighboring cells. The interference can vary due to other reasons as well as the geographical area, weather conditions, and user location. However, the authors did not consider any of these parameters to avoid the interference.

Fractional frequency reuse (FFR) is used in [15] where the frequency band is divided into several sub-bands to avoid the interference. The authors consider the FRF one for cell center users and three for cell edge users. This method allocates different sub-bands for different cell edge areas. In [16], a method using frequency reuse and pilot sensing is proposed to mitigate the cross-tier co-channel interference. After deploying the FRF of three to the macrocell, the femtocell senses the pilot signals from the adjacent cell. The scheduler then allocates the remaining frequency to the femtocell, which has not been allocated to another cell.

In [17], authors consider reducing the interference as a Gaussian random variate. They proposed three RB allocation techniques, namely, pure random allocation, compact random allocation and allocation with memory. The authors in [18] present an interference avoidance method to prevent strong inter-cell interference for the cell-edge user. Their proposed method consists of a two-tier algorithm - the first one at the eNB level and the second one at a central controller. They consider this controller as a group of neighboring eNBs that are connected to each other. According to their model, users send channel state information (CSI) with the interference of two sectors, whereas sectors send restriction request to the central controller. The controller then resolves the conflicts of the request and sends modified restriction lists to all involved eNBs. A downlink optimal power allocation scheme is used in [19] to maximize the spectral efficiency of the cell-edge user.

The MLWDF scheduling algorithm has been studied in [20-22] to analyze the performance and maximize the QoS of cell user. A delay priority based algorithm is used in [20] where the user gets higher priority if the packet delay is close to the threshold for real-time data. However, the authors do not consider the non-real-time data packets. The authors in [21] show that MLWDF provides better QoS and Quality of Experience (QoE) than other scheduling algorithms in terms of packet loss ratio and delay. In [22], an MLWDF based joint scheduling has been proposed with multiple traffic classes, where the priority of traffic class and waiting time in the queue of each packet are considered. Although the authors assume that the average SINR for each user is the same, they also ignore the Channel Quality Indicator (CQI) feedback. 


\section{System Model}

MLWDF is a throughput-optimal scheduling algorithm that considers HOL packet delay, the probability of packet loss and delay threshold along with instantaneous and previous throughput of the user. The scheme is defined by the following Equation (1):

$$
m_{i, j}{ }^{M L W D F}=\frac{-\log \delta_{i}}{\tau_{i}} \cdot D_{H O L, i} \cdot \psi_{i, j}
$$

where

$$
\psi_{i, j}=\frac{r_{i}(t)}{r_{i}(t-1)} .
$$

Here, $D_{H O L, i}$ denotes HOL packet delay, which is the waiting time between the packet arrival time and the time it is transmitted successfully. $\delta_{i}$ represents the probability of packet loss and $\tau_{i}$ is the value of delay threshold. The delay threshold $\tau_{i}$ for user $i$ is based on user applications. Table 1 shows the threshold values with priority for different types of data. Here, voice has the highest priority and (Transmission Control Protocol) TCP-based service has the lowest priority. Therefore, the data packet of TCP-based service would be discarded first in a congested queue.

Table 1. Delay threshold value for different types of data [23].

\begin{tabular}{ccc}
\hline Types of Data & Delay Threshold $(\mathbf{m s})$ & Priority \\
\hline Gaming & 50 & 3 \\
Conversational Voice & 100 & 2 \\
Live Video streaming & 100 & 7 \\
TCP-based (HTTP, FTP) & 300 & 8 \\
\hline
\end{tabular}

TCP: Transmission Control Protocol; HTTP: HyperText Transfer Protocol; FTP: File Transfer Protocol.

The scheduler maximizes the utility function $r_{i}(t) / r_{i}(t-1)$ for better system performance, which can be defined as weighted transmission rate $\psi_{i, j}$ in Equation (2). Here, $r_{i}(t)$ is the instantaneous transmission rate of user $i$ at transmission time interval (TTI) $t$ and $r_{i}(t-1)$ is the previous average transmission rate. Time $t$ is removed from the rest of the paper to reduce the complexity. For convenience, Table 2 shows the symbols used in this paper.

Table 2. Symbols used in the paper.

\begin{tabular}{cc}
\hline Symbol & Definition \\
\hline$i$ & user \\
$j$ & RB \\
$r_{i}$ & time (TTI index) \\
$D_{H O L, i}$ & instantaneous transmission rate of user $i$ \\
$\delta_{i}$ & HOL Delay of user $i$, RB $j$ \\
$\tau_{i}$ & probability of packet loss of user $i$ \\
$\Omega$ & delay threshold of user $i$ \\
$R_{i}$ & radius of the inner region \\
$S_{i}$ & distance from the eNB to user $i$ \\
$\varepsilon_{i}$ & probability from failure rate to success rate of user $i$ \\
$\gamma_{i, j}$ & the error probability of user $i$ \\
$G_{i, j}$ & received SINR of user $i$, RB $j$ \\
$P_{i, j}$ & channel gain from serving eNB of user $i, \mathrm{RB} j$ \\
$I$ & transmit power of serving eNB for user $i, \mathrm{RB} j$ \\
$\sigma$ & inter-cell interference \\
\hline
\end{tabular}

TTI: Transmission Time Interval; HOL: Head of Line; eNB: eNodeB; SINR: Signal to Interference plus Noise Ratio.

This paper defines the QoS requirement of the user by considering the HOL delay. The delays of the users with different applications must be kept below a predefined value for successful packet transmission. Therefore, the QoS requirement can be defined as follows: 


$$
\operatorname{Pr}\left\{D_{H O L, i}>\tau_{i}\right\} \leq \delta_{i}
$$

Equation (3) states that the probability of HOL delay of exceeding the delay threshold must be equal to or less than the probability of packet loss. This algorithm gives the priority to the user who has high HOL delay to avoid the packet loss. Thus, it can significantly improve the throughput and can serve more users with their required QoS.

SINR generally decreases when a user is moving far away from the eNB. Therefore, cell-edge users with lower SINR experience relatively lower throughput and higher packet loss. However, in different cases, the SINR value might be changed due to the weather conditions, geographical area, infrastructure or various types of noises. The MLWDF based proposed model divides the cell area into two different regions: inner and outer. The reason behind this is to separate the cell-edge and cell-centered user and to give more priority to the cell-edge users who are in the outer region by allocating more RBs to them. The distance between user and the attached eNB is considered along with SINR for accurate classification of the user. The maximum length from the eNB to the edge of inner region is $\Omega$. Any user who is located outside this area will be treated as a cell-edge user and the rest of the user will be considered as a cell-centered user. The proposed EMLWDF algorithm is explained in Equation (3) below:

$$
m_{i, j} E M L W D F=\frac{-\log \left(\frac{\delta_{i}}{W_{i}}\right)}{\tau_{i}} \cdot D_{H O L, i} \cdot \psi_{i, j},
$$

where

$$
\begin{gathered}
W_{i}=\left\{\begin{array}{ccc}
\frac{R_{i}}{\gamma_{i, j}} & \text { if } \quad R_{i}>\Omega \\
1 & \text { if } \quad R_{i} \leq \Omega
\end{array},\right. \\
R_{i}=\sqrt{\sum_{k=1}^{n}\left|x_{k}-y_{k}\right|^{2}}, \\
\gamma_{i, j}=\frac{G_{i, j} P_{i, j}}{\sigma^{2}+I} .
\end{gathered}
$$

Here, $m_{i, j} E M L W D F$ denotes the metric value of the EMLWDF algorithm, $\gamma_{i, j}$ is the SINR of user $i$ RB $j$ and $R_{i}$ is the distance between eNB and user $i$. The higher the metric $m_{i, j}$ EMLWDF value for a particular user in Equation (4), the higher the chance for that user to get the required RBs. Based on Equations (4) and (5), it can be seen that the variable $W_{i}$ will vary according to the location of the user $i$. If the distance of the user $i$ from eNB is more than the radius of the inner region $\Omega$, the proposed EMLWDF algorithm will consider the distance $R_{i}$ and SINR $\gamma_{i, j}$ of user $i \operatorname{RB} j$ to calculate the metric $m_{i, j} E M L W D F$. Otherwise, the user will be considered as a cell-centered user and the EMLWDF algorithm will not consider the distance and SINR of that particular user. After exceeding the inner region value, the higher the distance from eNB and the less SINR of the user, the higher the metric value and thus higher the possibility to get RBs.

The distance between the eNB position $x$ and user position $y$ with $n$ dimensions is measured in Equation (6) by the Pythagorean theorem considering Euclidian space [24]. Equation (7) calculates the received SINR $\gamma_{i, j}$ of user $i$ on $\mathrm{RB} j$, where $G_{i, j}$ and $P_{i, j}$ are the channel gain and transmit power of serving eNB, respectively [25]. $\sigma^{2}$ is the white noise power spectral density and $I$ is the inter-cell interference. The users are affected by various noise and interference in the real-life wireless environment. Large scale errors occur when the users are receiving the data packets. Therefore, this paper implements the two-state Markov Model to measure the probability of packet loss $\delta_{i}$ [26]. Success state and failure state are the two states in this model. The success state has low error probability and that helps to transmit packet successfully. On the other hand, the failure state has higher error probability. Thus, packets cannot be transmitted, which makes this state unusable. If the packet transmission fails, the packet is retransmitted until it exceeds the delay threshold value. After exceeding the threshold value, the packet will be discarded and the next packet transmission will begin. The packet-dropping operation 
has a significant impact on the probability of packet loss. This paper considers that the success state is the usable system scenario as packets can be transmitted successfully in this state. The delay threshold and error probability are considered in the simplified equation of success state, which is described in the following Equation (8):

$$
\delta_{i}=\varepsilon_{i}\left(1-S_{i}\right)^{\tau_{i}}
$$

Here, $\varepsilon_{i}$ is the error probability and $S_{i}$ is the probability from failure state to success state. The $\varepsilon_{i}$ is assumed as a small value and $\varepsilon_{i}$ should be smaller than $S_{i}$ in this state to make the system usable. According to [26], $\delta_{i}$ can be measured precisely by assuming a constant value of $\varepsilon_{i}$ and $S_{i}$ along with changing the delay threshold value $\tau_{i}$. Therefore, this paper considers this for success state $\varepsilon_{i}=0.01$, $S_{i}=0.1$ and $\tau_{i}$ based on the different user applications. The $\varepsilon_{i}$ is comparatively larger than $S_{i}$ in the failure state due to the high error probability and $\delta_{i}$ can be defined as $\delta_{i} \approx 1 \approx \varepsilon_{i}$, which can make the system unusable. Considering the error probability and the probability from failure state to success state in Equation (8) and after doing simplification, the Equation (4) can be written as follows:

$$
\begin{gathered}
m_{i, j} E M L W D F=\frac{-\log \left(\frac{\varepsilon_{i}\left(1-S_{i}\right)^{\tau_{i}}}{W_{i}}\right)}{\tau_{i}} \cdot D_{H O L, i} \cdot \psi_{i, j}, \\
m_{i, j} E M L W D F=\left\{\frac{-\log \varepsilon_{i}-\tau_{i} \log \left(1-S_{i}\right)+\log W_{i}}{\tau_{i}}\right\} \cdot D_{H O L, i} \cdot \psi_{i, j}, \\
m_{i, j} E M L W D F=\left\{\frac{1}{\tau_{i}} \log \left(\frac{W_{i}}{\varepsilon_{i}}\right)-\log \left(1-S_{i}\right)\right\} \cdot D_{H O L, i} \cdot \psi_{i, j}
\end{gathered}
$$

The following Figure 2 shows the step-by-step procedure of the proposed Extended MLWDF (ELMLWDF) algorithm.

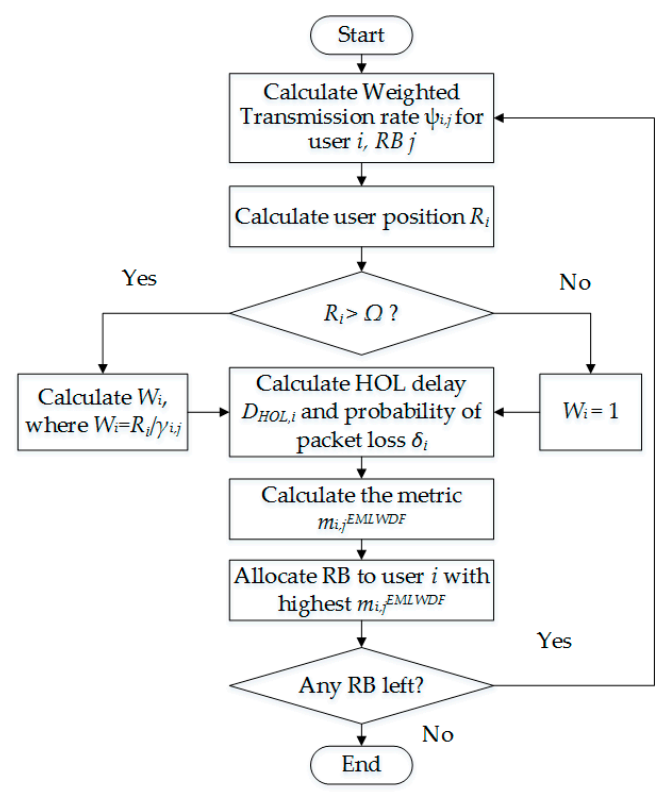

Figure 2. Step-by-step procedure of the proposed method.

\section{Results and Discussion}

The MATLAB based Vienna LTE System level simulator is used to measure the performance of the proposed model [27]. The downlink channel model with microscopic path loss and shadow fading for an urban area is considered in the simulation process. The WINNER II channel model is implemented in the MATLAB based simulator [28]. Users are equally distributed and scattered randomly in the urban area. There are a different number of users in each cell, which varies from 10 to 60 . The users are 
not static and their average speed is $5 \mathrm{kmph}$. A $900 \mathrm{MHz}$ frequency band with $20 \mathrm{MHz}$ bandwidth is used and simulation time is 1000 TTI. A number of simulations have been conducted to evaluate the cell-edge throughput, average user throughput, spectral efficiency and fairness index of the proposed EMLWDF method, and the results are compared with PF and basic MLDWF schedulers.

Three types of data are considered in the simulation namely Video, Voice over Internet Protocol (VoIP), and HyperText Transfer Protocol (HTTP). The resource type of VoIP is considered as guaranteed bit rate (GBR), whereas Video and HTTP are considered as non-guaranteed bit rate (NGBR) for all user. In order to provide QoS guaranteed VoIP performance, an RTP (Real-time Transport Protocol) AMR (Adaptive Multi-Rate) 12.2 codec with a $12.2 \mathrm{kbps}$ bitrate is used [29]. Videos are encoded by an H.264/AVC (Advanced Video Coding) encoder with a constant bitrate of $242 \mathrm{kbps}$, while this paper considers the bitrate of $250 \mathrm{kbps}$ for HTTP packets [9]. The basic parameters of the simulation are stated in Table 3.

Table 3. Simulation parameters with corresponding values.

\begin{tabular}{cc}
\hline Parameters & Values \\
\hline System bandwidth & $20 \mathrm{MHz}$ \\
Operating frequency & $900 \mathrm{MHz}$ \\
Scenario & Random deployment (Urban) \\
Number of users & $10,20,30,40,50,60$ \\
User speed & $5 \mathrm{kmph}$ \\
eNB power transmission & $46 \mathrm{dBm}$ \\
MSC index & 29 available MSCs as in 3GPP [30] \\
Traffic model & Video, VoIP, HTTP \\
Scheduler & PF, MLWDF, EMLWDF \\
Simulation time & 1000 TTI \\
\hline
\end{tabular}

MSC: Modulation Coding Scheme; 3GPP: Third Generation Partnership Project; VoIP: Voice over Internet Protocol; PF: Proportional fairness; MLWDF: Modified Largest Weighted Delay First; EMLWDF: Extended MLWDF.

The cell-edge throughput of the user is calculated for different data types and algorithms with a various number of users. These cell-edge users are located in the outer region of the cell and their distances from the eNB are higher than the value of $\Omega$. Figures 3-5 show the comparison of PF, basic MLWDF and EMLWDF algorithms for video, VoIP and HTTP, respectively. As predicted, when the number of users is increasing, the cell-edge user throughput is decreasing for all algorithms. The proposed EMLWDF has higher cell-edge throughput than the other two algorithms for all types of user data. The reasons for this better performance are (a) EMLWDF considers the distance between the user and eNB, received SINR of the user along with HOL delay and previous throughput. Therefore, the users located in a cell-edge area with bad channel condition can get priority from the scheduler and receive a comparatively higher number of RBs.

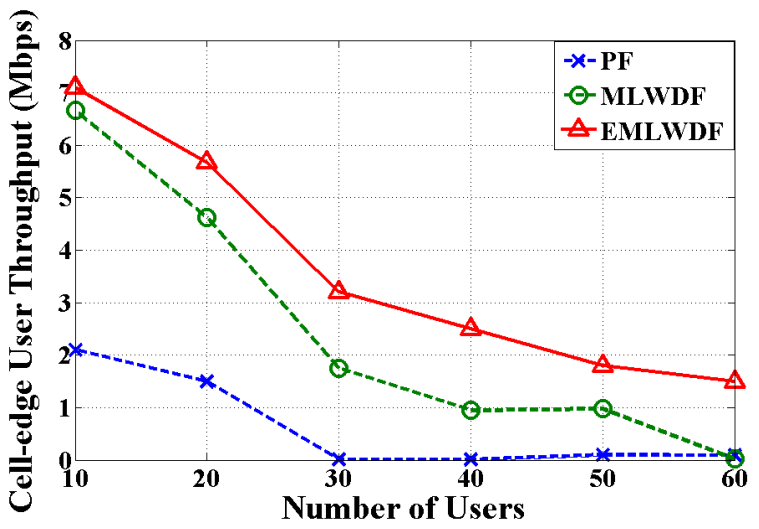

Figure 3. Cell-edge user throughput for Video. 


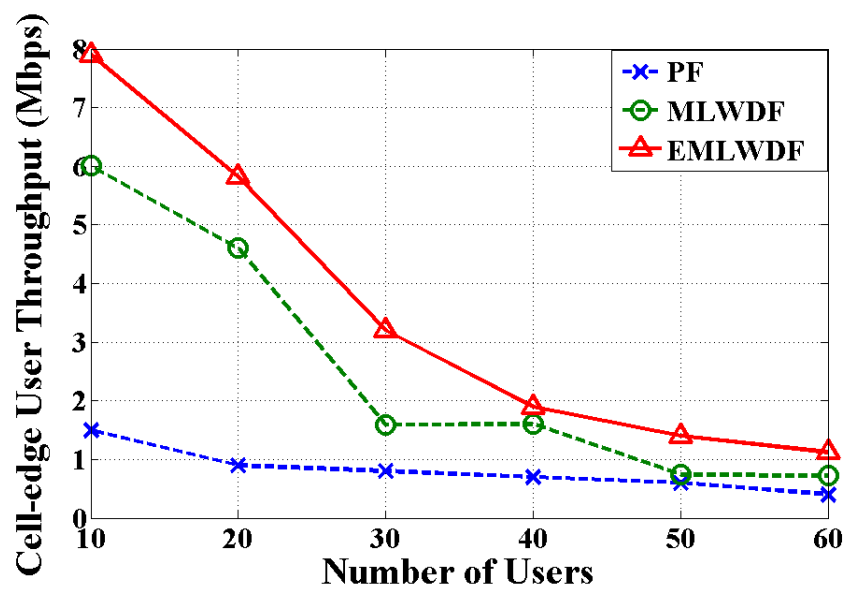

Figure 4. Cell-edge user throughput for VoIP.

On the other hand, basic MLWDF does not consider any other parameter rather than HOL delay and previous throughput. As a result, the cell-edge users do not get the required RB, whereas cell-edge user throughput of the PF algorithm is the lowest, as it only considers the previous throughput of the user. Furthermore, (b) EMLWDF measures the probability of packet loss by considering the error probability of data packet and probability from failure state to success state. Therefore, the cell-edge users with higher error rates are getting higher priority from the scheduler to get more RB. However, the other two algorithms are experiencing relatively higher packet loss as they do not consider these parameters that cause lower cell-edge throughput.

Figures 3-5 also show that EMLWDF outperforms the other algorithms for all types of user data. The proposed EMLWDF is on average $88.59 \%$ and $46.04 \%$ better than PF and MLWDF algorithms regarding cell-edge throughput for Video type data. Moreover, EMLWDF increases the cell-edge throughput by $72.17 \%$ and $39.33 \%$ for VoIP and $77.30 \%$ and $13.93 \%$ for HTTP types of data compared with the PF and MLWDF algorithms, respectively. Table 4 shows the average improvement of proposed EMLWDF for different types of data.

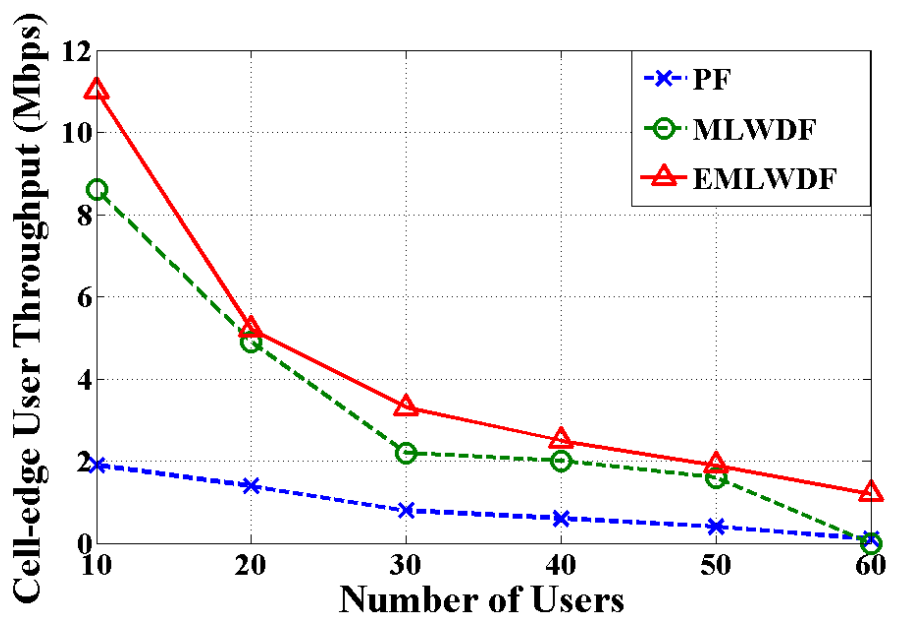

Figure 5. Cell-edge user throughput for HTTP.

Average user throughput is calculated by considering all the users of the cell area to demonstrate that the proposed EMLWDF algorithm is not only efficient for the cell-edge user but also for all other users of the cell. Figures 6-8 show the average user throughput of different algorithms for three different data types. All of the figures show that the EMLWDF algorithm achieves better average user 
throughput than the other two algorithms. Although EMLWDF has marginal improvements for VoIP type data in Figure 6, it still performs $31.80 \%$ and $4.86 \%$ better than PF and MLWDF, respectively. The reasons for this higher user throughput are (a) the EMLWDF algorithm considers the distance and the received SINR only when the users are located outside the inner region. Otherwise, it distributes the RBs among the users based on HOL delay and throughputs. Therefore, all users get the required RBs and can maintain the throughput. In addition, (b) the EMLWDF algorithm also ensures that other users in the cell center area do not starve for RBs due to prioritizing the cell-edge user by taking the logarithm expression of the distance and received SINR rather than direct implementation. Thus, the cell-edge users will not be allocated to all of the available RBs and the scheduler can serve the cell center users according to their requirements.

Table 4. Improvement comparison of proposed EMLWDF method.

\begin{tabular}{cccc}
\hline Traffic Model & Algorithm & Cell-Edge User Throughput (\%) & Average User Throughput (\%) \\
\hline \multirow{2}{*}{ Video } & PF & 88.59 & 27.23 \\
& MLWDF & 46.04 & 11.57 \\
\hline \multirow{2}{*}{ VoIP } & PF & 72.17 & 31.80 \\
& MLWDF & 39.33 & 4.86 \\
\hline \multirow{2}{*}{ HTTP } & PF & 77.30 & 44.71 \\
& MLWDF & 13.93 & 19.34 \\
\hline
\end{tabular}

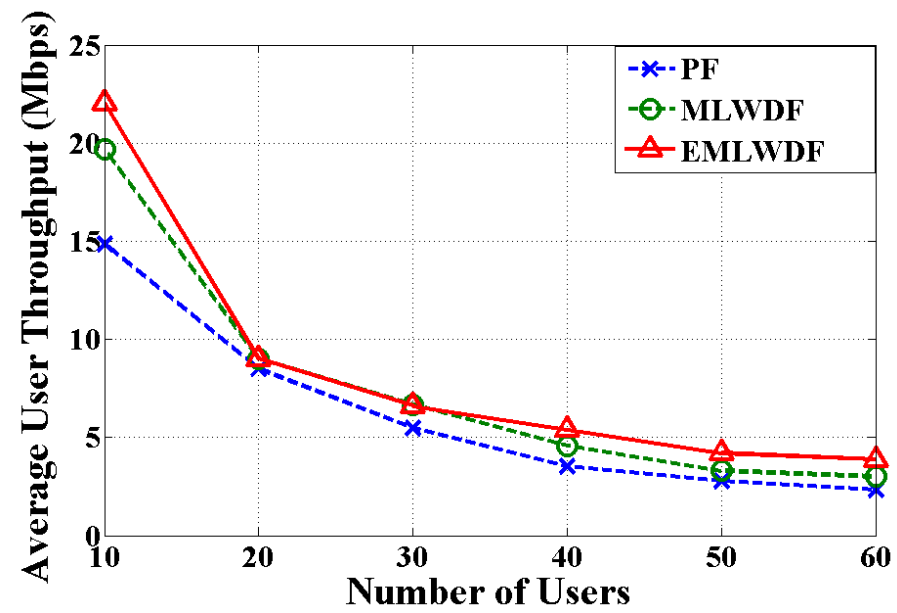

Figure 6. Average user throughput for Video.

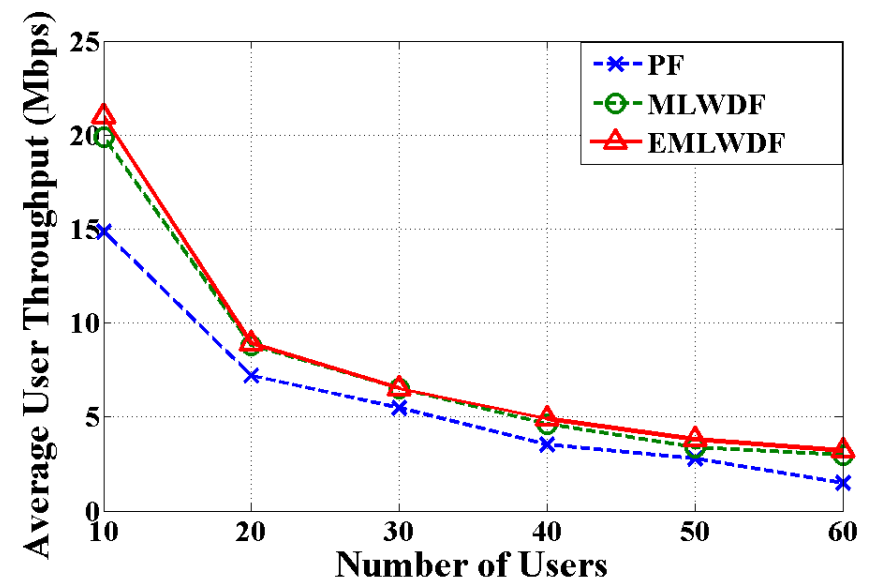

Figure 7. Average user throughput for VoIP. 


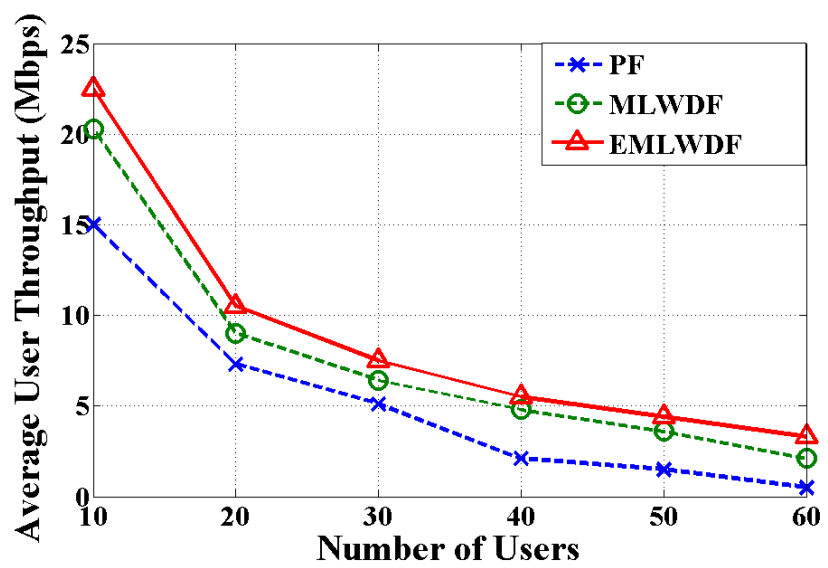

Figure 8. Average user throughput for HTTP.

The fairness index of the user is calculated by the Jain's fairness index equation [31], which can be described as, $F=\left(\sum_{i=1}^{n} \beta_{i}\right)^{2} / n \sum_{i=1}^{n} \beta_{i}^{2}$. Here, $F$ is the fairness index, $\beta_{i}$ is the average throughput of user $i$, and $n$ denotes the number of users. The fairness index $F$ ranges from $1 / n$ to 1 . Mixed data types for all users in the cell are considered to calculate the fairness. Figure 9 shows the comparison of the fairness index among different algorithms with a various number of users. It is clear from this comparison that EMLWDF performs better than the other algorithms. EMLWDF has $43.77 \%$ and $4.66 \%$ better fairness than PF and MLWDF, respectively. By considering the distance of the user and received SINR along with HOL delay and the probability of packet loss, the proposed EMLWDF distributes RBs more efficiently to all users based on their requirements. The users with high priority application and higher delay along with cell-edge users get the higher priority in this method to get the RBs, which enhances the throughput as well as fairness index. Therefore, the EMLWDF algorithm can satisfy the users with GBR by providing the required RBs and at the same time satisfy more users with NGBR compared to other algorithms by allocating RBs efficiently. The fairness index of the MLWDF algorithm is close to the EMLWDF algorithm due to the channel and QoS awareness. However, the $\mathrm{PF}$ algorithm allocates RBs to the user without considering the distance of the user from eNB and the probability of packet loss. Furthermore, PF is the only channel aware but QoS-unaware algorithm in this comparison that does not consider the QoS requirement of the user. Thus, PF cannot fully satisfy the user with strict delay threshold, which leads to high packet loss and low throughput. Therefore, considering all of these facts, PF has the lowest fairness in this comparison. The figure also shows that the fairness index decreases as the number of users increases due to the limitation of the available RBs in the cell.

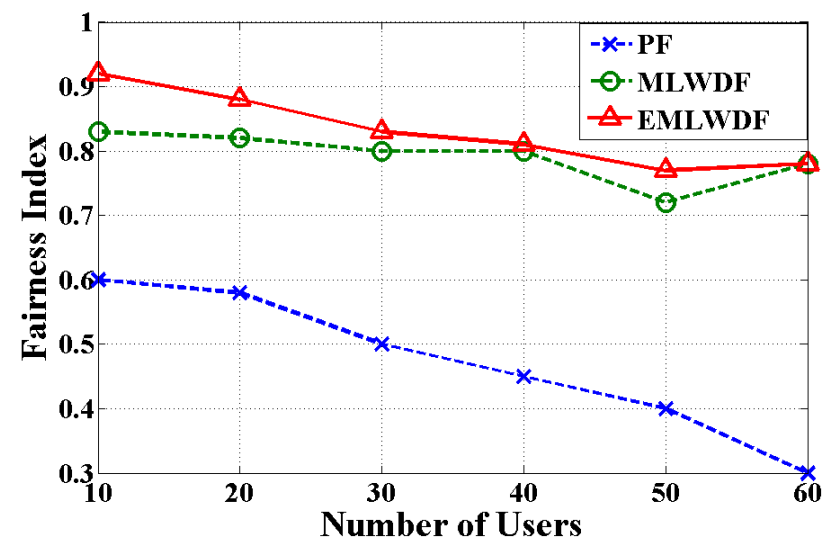

Figure 9. Fairness index for different algorithms. 
Spectral efficiency is calculated by the ratio of the total bits of all users successfully transferred and the total bandwidth of the channel for a specific time. Thus, the unit of spectral efficiency is bit/s/Hz. The spectral efficiency comparison in Figure 10 demonstrates that EMLWDF utilizes the spectrum more efficiently than PF and MLWDF. Ensuring the adequate distribution of the RBs by EMLWDF avoids the waste of spectrum resources and thus increases the efficiency. On the contrary, PF only considers current and previous data rates, whereas MLWDF uses delay, which leads to lower spectral efficiency. Overall, $33.31 \%$ and $3.53 \%$ improvement have been achieved by the proposed EMLWDF compared to MLWDF and PF algorithms, respectively. This paper implements $900 \mathrm{MHz}$ as the operating frequency. Figure 11 shows a cell-edge throughput comparison by implementing the EMLWDF algorithm in $900 \mathrm{MHz}, 1800 \mathrm{MHz}$ and $2100 \mathrm{MHz}$ frequency bands with the same bandwidth for VoIP data. The $900 \mathrm{MHz}$ band achieves $5.23 \%$ and $4.0 \%$ higher cell-edge throughput than $1800 \mathrm{MHz}$ and $2100 \mathrm{MHz}$ bands, respectively. Important to note here is that both $1800 \mathrm{MHz}$ and $2100 \mathrm{MHz}$ frequency bands still perform better than the PF and MLWDF algorithms that are implemented in this paper. Figure 12 shows the effect of the user speed on the cell-edge user throughput for EMLWDF algorithm. It is clear that the static users have throughput than other users. The users with $5 \mathrm{kmph}$ speed have $4.85 \%$ less cell-edge throughput and the users with $40 \mathrm{kmph}$ speed have $42.36 \%$ less than the static users due to the higher packet loss, longer delay, and low SINR.

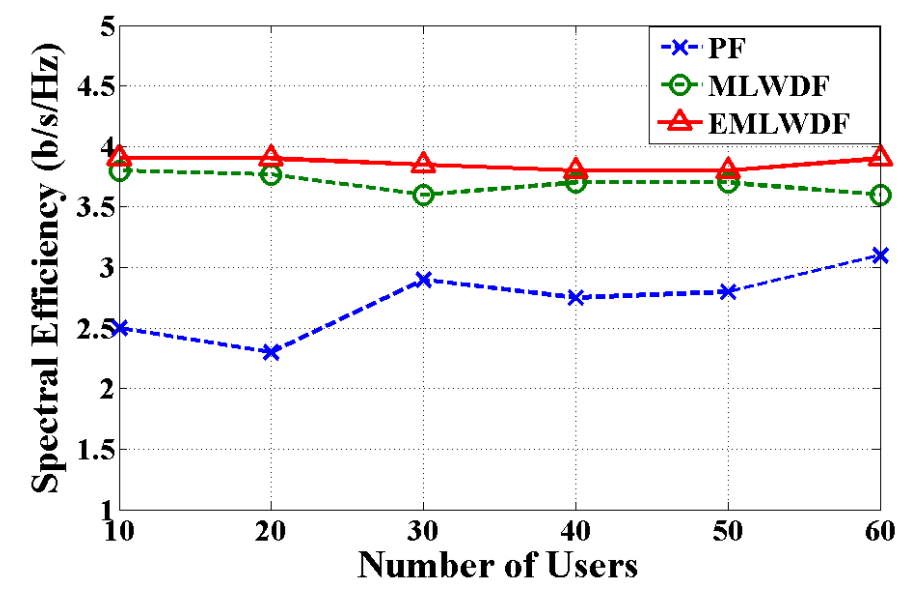

Figure 10. Spectral efficiency of different algorithms.

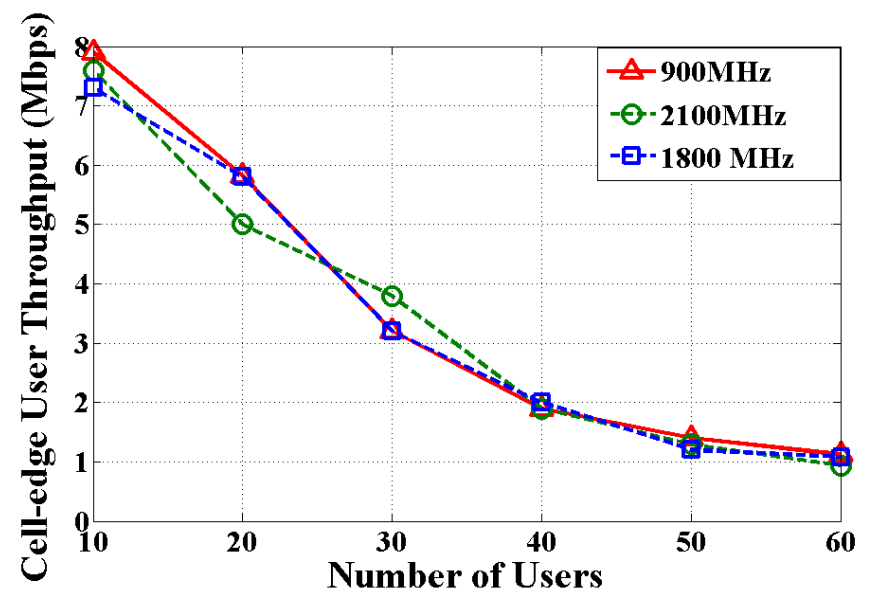

Figure 11. Comparison of different frequencies with EMLWDF for VoIP. 


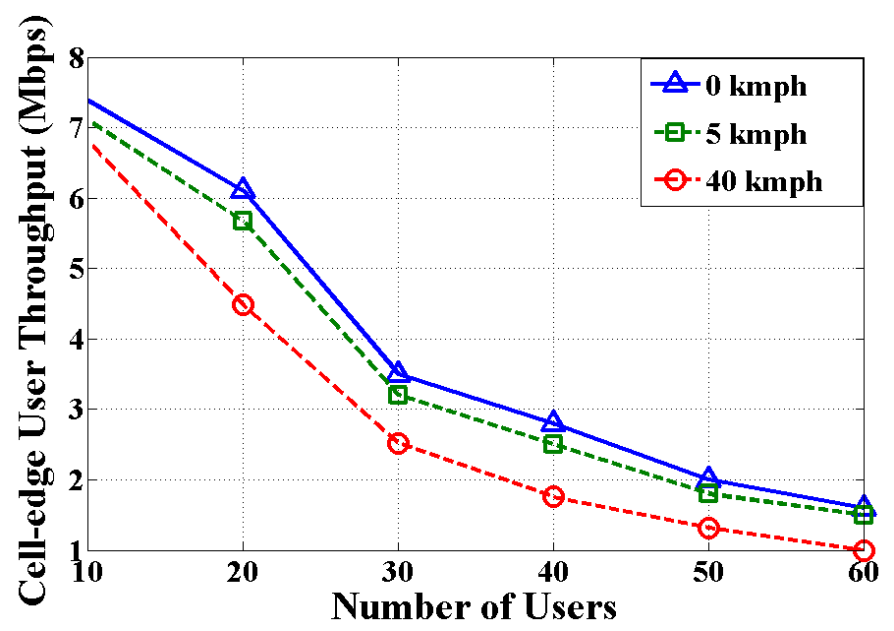

Figure 12. Comparison of different user speeds with EMLWDF for Video.

\section{Limitation and Future Works}

The main objective of the proposed EMLWDF algorithm is to enhance the QoS of cell-edge users along with maintaining the average user throughput. However, the performance of the proposed algorithm decreases when there are a higher number of users in the cell due to the lack of available resources and high congestion. As a future work, the above problem can be solved by introducing the queue length of each user to the scheduler for different types of data. Queue length for real-time and non-real-time data will help the scheduler to allocate the RBs to the users according to their requirements more efficiently. Moreover, implementing improved and higher multiple input multiple output (MIMO) techniques can increase the capacity of the cell and thus can serve more users.

\section{Conclusions}

This paper has proposed an EMLWDF scheduling algorithm for the LTE network to enhance the QoS of cell-edge users. This modified algorithm considers the distance of the user from eNB, received SINR of the user, error probability and the probability from failure rate to success rate to give more priority to the users who are far away from the eNB. The distance and SINR parameters are considered with the basic MLWDF algorithm only if the users are located outside the inner region of the cell. Otherwise, the proposed EMLWDF works the same as the basic MLWDF to ensure that cell-centered users have also been allocated to the required RBs. Simulation results for three different data types show that not only has the cell-edge user throughput increased, but the average user throughput has also increased $23.25 \%$ overall compared to the PF and MLWDF algorithms. Moreover, the proposed EMLWDF demonstrates a significant improvement in the fairness index and in spectral efficiency.

Acknowledgments: This research work is supported by the scheme no. PV001-2014 of the Centre of Research Grant Management (PPGP), University of Malaya, Malaysia.

Author Contributions: H.R. Chayon and H. Ramiah conceived and designed the experiments; H.R. Chayon performed the experiments; H.R. Chayon, A.W. Reza and K. Dimyati analyzed the data; H. Ramiah, K. Dimyati and A.W. Reza contributed reagents/materials/analysis tools; H.R. Chayon wrote the paper.

Conflicts of Interest: The authors declare no conflict of interest. The funding sponsors had no role in the design of the study; in the collection, analyses, or interpretation of data; in the writing of the manuscript, and in the decision to publish the results.

\section{References}

1. Nagate, A; Ogata, D.; Fujii, T. Cell Edge Throughput Improvement by Base Station Cooperative Transmission Control with Reference Signal Interference Canceller in LTE System. In Proceedings of the IEEE 75th Vehicular Technology Conference (VTC Spring), Yokohama, Japan, 6-9 May 2012; pp. 1-5. 
2. Capozzi, F.; Piro, G.; Grieco, L.A.; Boggia, G.; Camarda, P. Downlink packet scheduling in LTE cellular networks: Key design issues and a survey. IEEE Commun. Surv. Tutor. 2013, 15, 678-700. [CrossRef]

3. Evolved Universal Terrestrial Radio Access (E-UTRA) and Evolved Universal Terrestrial Radio Access Network (E-UTRAN). Overall Description; Stage 2; TS 36.300; 3rd Generation Partnership Project (3GPP): Valbonne, France, 2012.

4. Evolved Universal Terrestrial Radio Access (E-UTRA). Physical Channels and Modulation; TS 36.211; 3rd Generation Partnership Project (3GPP): Valbonne, France, 2012.

5. Choi, J.-G.; Bahk, S. Cell-throughput analysis of the proportional fair scheduler in the single-cell environment. IEEE Trans. Veh. Technol. 2007, 56, 766-778. [CrossRef]

6. Andrews, M.; Kumaran, K.; Ramanan, K.; Stolyar, A.; Whiting, P.; Vijayakumar, R. Providing quality of service over a shared wireless link. IEEE Commun. Mag. 2001, 39, 150-154. [CrossRef]

7. Stolyar, A.L.; Ramanan, K. Largest weighted delay first scheduling: Large deviations and optimality. Ann. Appl. Probab. 2001, 11, 1-48. [CrossRef]

8. Basukala, R.; Ramli, H.M.; Sandrasegaran, K. Performance analysis of EXP/PF and M-LWDF in downlink 3GPP LTE system. In Proceedings of the First Asian Himalayas International Conference on Internet (AH-ICI), Kathmandu, Nepal, 3-5 November 2009; pp. 1-5.

9. Singh, D. Performance Analysis of QOS-aware Resource Scheduling Strategies in LTE Femtocell Networks. Int. J. Eng. Trends Technol. 2013, 1, 2994-2999.

10. Boudreau, G.; Panicker, J.; Guo, N.; Chan, R.; Wang, N.; Vrzic, S. Interference coordination and cancellation for $4 \mathrm{G}$ networks. IEEE Commun. Mag. 2009, 47, 74-81. [CrossRef]

11. Himayat, N.; Talwar, S.; Rao, A.; Soni, R. Interference management for $4 \mathrm{G}$ cellular standards [WIMAX/LTE UPDATE]. IEEE Commun. Mag. 2010, 48, 86-92. [CrossRef]

12. Lee, D.; Li, G.Y.; Tang, S. Intercell interference coordination for LTE systems. IEEE Trans. Veh. Technol. 2013, $62,4408-4420$.

13. Zhang, X.; He, C.; Jiang, L.; Xu, J. Inter-cell interference coordination based on softer frequency reuse in OFDMA cellular systems. In Proceedings of the International Conference on Neural Networks and Signal Processing, Nanjing, China, 7-11 June 2008; pp. 270-275.

14. Qian, M.; Hardjawana, W.; Li, Y.; Vucetic, B.; Shi, J.; Yang, X. Inter-cell interference coordination through adaptive soft frequency reuse in LTE networks. In Proceedings of the IEEE Wireless Communications and Networking Conference (WCNC), Shanghai, China, 1-4 April 2012; pp. 1618-1623.

15. Lee, P.; Lee, T.; Jeong, J.; Shin, J. Interference management in LTE femtocell systems using fractional frequency reuse. In Proceedings of the 12th International Conference on Advanced Communication Technology (ICACT), Gangwon-Do, Korea, 7-10 February 2010; pp. 1047-1051.

16. Kim, T.-H.; Lee, T.-J. Throughput enhancement of macro and femto networks by frequency reuse and pilot sensing. In Proceedings of the IEEE International Performance, Computing and Communications Conference (IPCCC), Austin, TX, USA, 7-9 December 2008; pp. 390-394.

17. Risi, C.; Wassie, D.A. Inter-cell interference modeling in LTE systems. Wirel. Pers. Commun. 2013, 72, 389-404. [CrossRef]

18. Rahman, M.; Yanikomeroglu, H. Enhancing cell-edge performance: A downlink dynamic interference avoidance scheme with inter-cell coordination. IEEE Trans. Wirel. Commun. 2010, 9, 1414-1425. [CrossRef]

19. Chaudhuri, S.; Pradeep, K.; Das, D. Maximizing spectral efficiency for cell edge users in LTE small cell network. In Proceedings of the IEEE International Conference on Communications and Signal Processing (ICCSP), Melmaruvathur, India, 3-5 April 2014; pp. 1312-1317.

20. Li, Y.P.; Hu, B.J.; Zhu, H.; Wei, Z.H.; Gao, W. A delay priority scheduling algorithm for downlink real-time traffic in LTE networks. In Proceedings of the IEEE Information Technology, Networking, Electronic and Automation Control Conference, Chongqing, China, 20-22 May 2016; pp. 706-709.

21. Park, W.-H.; Cho, S.; Bahk, S. Scheduler design for multiple traffic classes in OFDMA networks. Comput. Commun. 2008, 31, 174-184. [CrossRef]

22. Alfayly, A.; Mkwawa, I.; Sun, L.; Ifeachor, E. QoE-based performance evaluation of scheduling algorithms over LTE. In Proceedings of the IEEE Globecom Workshops (GC Wkshps), Anaheim, CA, USA, 3-7 December 2012; pp. 1362-1366.

23. General Packet Radio Service (GPRS). Enhancements for Evolved Universal Terrestrial Radio Access Network (E-UTRAN) Access; TS 23.401 V10.6.0; 3rd Generation Partnership Project (3GPP): Valbonne, France, 2011. 
24. Abbena, E.; Salamon, S.; Gray, A. Modern Differential Geometry of Curves and Surfaces with Mathematica; CRC Press: Boca Raton, FL, USA, 2006.

25. Chand, P.; Mahapatra, R.; Prakash, R. Energy efficient radio resource management for heterogeneous wireless network using CoMP. Wirel. Netw. 2016, 22, 1093-1106. [CrossRef]

26. Lee, K.K.; Chanson, S.T. Packet loss probability for real-time wireless communications. IEEE Trans. Veh. Technol. 2002, 51, 1569-1575. [CrossRef]

27. Institute of Telecommunications, Vienna University of Technology. LTE System Level Simulator, v1.6 r885. Available online: http:/ / www.cs.odu.edu/ rnagella/LTE-simulation/LTEsystemDoc.pdf (accessed on 15 July 2016).

28. Hentilä, L.; Kyösti, P.; Käske, M.; Narandzic, M.; Alatossava, M. MATLAB implementation of the WINNER Phase II. Channel Model ver1.1. Available online: http:/ / projects.celtic-initiative.org/winner+/phase_2_ model.html (accessed on 15 July 2016).

29. Lte Physical Layer Framework for Performance Verification; TSG-RAN1 R1-070674; 3rd Generation Partnership Project (3GPP): Valbonne, France, 2007.

30. Evolved Universal Terrestrial Radio Access (E-UTRA). Physical Layer Procedures; TS 36.213 V11.0.0; 3rd Generation Partnership Project (3GPP): Valbonne, France, September 2012.

31. Jain, R.; Chiu, D.-M.; Hawe, W.R. A Quantitative Measure of Fairness and Discrimination for Resource Allocation in Shared Computer System; Eastern Research Laboratory, Digital Equipment Corporation: Hudson, MA, USA, 1984; Volume 38.

(C) 2017 by the authors. Licensee MDPI, Basel, Switzerland. This article is an open access article distributed under the terms and conditions of the Creative Commons Attribution (CC BY) license (http:/ / creativecommons.org/licenses/by/4.0/). 